Homology, Homotopy and Applications, vol.9(1), 2007, pp.275-293

\title{
ON LOW-DIMENSIONAL HOMOLOGY IN CATEGORIES
}

\author{
TOMAS EVERAERT AND MARINO GRAN \\ (communicated by George Janelidze)
}

\begin{abstract}
The classical Stallings-Stammbach five-term exact sequence associated with a short exact sequence of groups is shown to exist in a general categorical context. This makes it possible to establish a new result relating low-dimensional homology and central series, which extends a well known result from the theory of groups.
\end{abstract}

\section{Introduction}

As remarked by Stallings in his article [30], the homology of a group is related to the lower central series of the group. The observation we would like to make in this article is that this fact essentially depends on some categorical properties of the category of groups, so that this relation still exists in any category having these specific properties.

The Fröhlich school developed a homology theory for varieties of $\Omega$-groups, allowing one to obtain, for instance, the invariance of the Hopf formula and the exactness of the Stallings-Stammbach sequence. Recall that a variety of $\Omega$-groups [19] is a variety whose theory contains the operations and identities of the theory of groups and each of whose $n$-ary operations $\omega \in \Omega$ satisfies the identity $\omega(e, e, \ldots, e)=e$, where $e$ is the unit of the group structure. Examples of $\Omega$-groups include varieties of groups, abelian groups, rings, commutative rings, associative algebras and Lie algebras. It is in this context that Fröhlich [14], Lue [27] and Furtado-Coelho [16] extended the following important results from the theory of groups.

Given a group $A$, and two free presentations $\frac{F}{R}$ and $\frac{F^{\prime}}{R^{\prime}}$ of $A$, there is an isomorphism

$$
\frac{R \wedge[F, F]}{[R, F]} \cong \frac{R^{\prime} \wedge\left[F^{\prime}, F^{\prime}\right]}{\left[R^{\prime}, F^{\prime}\right]}
$$

In fact, this is a consequence of Hopf's well known characterization of the second

T. Everaert's research is financed by a Ph.D. grant of the Institute of Promotion of Innovation through Science and Technology in Flanders (IWT-Vlaanderen).

Received March 10, 2006, revised January 8, 2007; published on February 12, 2007.

2000 Mathematics Subject Classification: 18G50, 20F14, 55N20.

Key words and phrases: homology, category theory, Hopf formula, central series.

Copyright (C) 2007, Tomas Everaert and Marino Gran. Permission to copy for private use granted. 
integral homology group

$$
H_{2}(G) \cong \frac{R \wedge[F, F]}{[R, F]}
$$

which is valid for any free presentation $A \cong \frac{F}{R}$.

A second result, due to Stallings $[\mathbf{3 0}]$ and Stammbach [31], states that any short exact sequence of groups

$$
0 \longrightarrow K \longrightarrow A \longrightarrow B \longrightarrow 0
$$

yields a five-term exact sequence

$$
H_{2}(A) \longrightarrow H_{2}(B) \longrightarrow \frac{K}{[K, A]} \longrightarrow H_{1}(A) \longrightarrow H_{1}(B) \longrightarrow 0,
$$

where 0 is the trivial group $\{e\}$.

Moreover, it was observed that all this remains valid when the subvariety subfunctor $\mathrm{Gp} \rightarrow \mathrm{Gp}$ sending a group $A$ to its derived subgroup $[A, A]$ is replaced by any term of the central series

$$
A \geqslant[A, A] \geqslant[[A, A], A] \geqslant[[[A, A], A], A] \geqslant \cdots .
$$

In [11], Everaert and Van der Linden extended these results from $\Omega$-groups to any semi-abelian category $[\mathbf{2 4}]$ with enough projectives.

Conduché and Ellis proved that $(\mathbf{A})$ and $(\mathbf{B})$ also hold in the category $B-$ $\operatorname{PrCM}$ of precrossed modules over a fixed group $B$, where the group commutator is now replaced by the Peiffer commutator [8]. It is noted that $B-\operatorname{PrCM}$ is not a semi-abelian category, simply because it is not pointed. These authors also proved a natural generalization of the following theorem from Stallings's article [30] for precrossed $B$-modules, which reveals a link between homology and central series.

Let $f: A \rightarrow B$ be a homomorphism of groups and assume that

1. the homomorphism $H_{1}(f): H_{1}(A) \rightarrow H_{1}(B)$ is an isomorphism;

2. the homomorphism $H_{2}(f): H_{2}(A) \rightarrow H_{2}(B)$ is surjective.

Then for any $n \geqslant 1$, the induced homomorphism $\frac{A}{A^{n}} \rightarrow \frac{B}{B^{n}}$ is an isomorphism, where $A^{n}$ (resp. $B^{n}$ ) is the $n$-th term in the lower central series of $A$ (resp. $B$ ).

In this article, we shall adopt an axiomatic approach, described in the first section, that enables all the above results to be unified. At the time when the Fröhlich school was investigating this subject, the categorical properties of groups that are essential in order to develop this theory had not all been discovered. Recent advances in categorical algebra $[\mathbf{3}, \mathbf{4}, \mathbf{5}, \mathbf{7}, \mathbf{2 1}, \mathbf{2 2}, \mathbf{2 4}, \mathbf{2 8}]$ now make it possible to build a more general theory based on simpler arguments.

\section{The setting}

In this section we recall some basic definitions and some useful properties of the categories we shall consider in the present article.

In this section we shall always assume that the category $\mathcal{A}$ is 
- finitely complete

- quasi-pointed

- regular

- protomodular.

Recall that a category $\mathcal{A}$ is quasi-pointed if it has an initial object 0 and a final object 1 , and the unique arrow $0 \rightarrow 1$ is a monomorphism. This implies that there is at most one arrow from an object $A$ to 0 ; when this is the case, it is necessarily a split epimorphism. A category $\mathcal{A}$ is regular if 1 ) any arrow has a (unique) factorization as a regular epimorphism followed by a monomorphism; 2) these factorizations are stable under pullbacks. In the regular epi-mono factorization $i \circ p: A \rightarrow I \rightarrow B$ of an arrow $f: A \rightarrow B$, the subobject $i: I \rightarrow B$ is called the regular "image" of the arrow $f$.

By kernel (respectively cokernel) of an arrow $f: A \rightarrow B$, we mean the pullback (respectively pushout) of that arrow along the initial arrow $0 \rightarrow B$ (along the arrow $A \rightarrow 0$, when this latter arrow exists). We write

$$
\operatorname{Ker}(f) \stackrel{\operatorname{ker}(f)}{\longrightarrow} A
$$

for the kernel of $f$ and

$$
A \stackrel{\operatorname{coker}(f)}{\longrightarrow} \operatorname{Coker}(f)
$$

for the cokernel of $f$. We say that a monomorphism $k: K \rightarrow A$ is normal, if $k$ is the kernel of some arrow.

A diagram

$$
A_{1} \stackrel{f_{1}}{\longrightarrow} A_{2} \stackrel{f_{2}}{\longrightarrow} A_{3}
$$

is called a short exact sequence if $f_{1}=\operatorname{ker}\left(f_{2}\right)$ and $f_{2}$ is a regular epimorphism. A diagram

$$
\cdots \longrightarrow A_{i-1} \stackrel{f_{i-1}}{\longrightarrow} A_{i} \stackrel{f_{i}}{\longrightarrow} A_{i+1} \longrightarrow \cdots
$$

is called an exact sequence if, for all $i$,

$$
I\left[f_{i-1}\right] \longrightarrow A_{i} \longrightarrow I\left[f_{i}\right]
$$

is a short exact sequence, where $I\left[f_{i-1}\right]$ and $I\left[f_{i}\right]$ are the regular images of the arrows $f_{i-1}$ and $f_{i}$, respectively. Note that a short exact sequence is not the same as an exact sequence

$$
0 \longrightarrow A_{1} \stackrel{f_{1}}{\longrightarrow} A_{2} \stackrel{f_{2}}{\longrightarrow} A_{3} \longrightarrow 0,
$$

simply because a morphism $A_{3} \rightarrow 0$ need not always exist. For example, $0 \rightarrow 1 \rightarrow 1$ is a short exact sequence, while a morphism $1 \rightarrow 0$ only exists if $\mathcal{A}$ is pointed.

When $\mathcal{A}$ is a quasi-pointed regular category, it is Bourn-protomodular [4] if and only if the Short Five Lemma holds in $\mathcal{A}$ : given any commutative diagram of short 
exact sequences

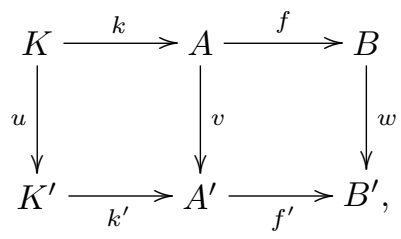

if $u$ and $w$ are isomorphisms, then $v$ is an isomorphism as well.

In a category $\mathcal{A}$ satisfying the conditions above, a regular epimorphism $p: A \rightarrow B$ is always the cokernel of its kernel and the notion of exact sequence defined above is just the usual one. As a consequence, the regular epi-mono factorization of an arrow $f: A \rightarrow B$ in $\mathcal{A}$ can be obtained in the following way: one first takes the kernel $\operatorname{ker}(f)$ of $f$, and then the cokernel $p: A \rightarrow I$ of $k e r(f)$. The induced factorization $i: I \rightarrow B$ is a monomorphism. The following property from [5] will be needed:

Proposition 2.1. Consider any commutative diagram of short exact sequences in $\mathcal{A}$ :

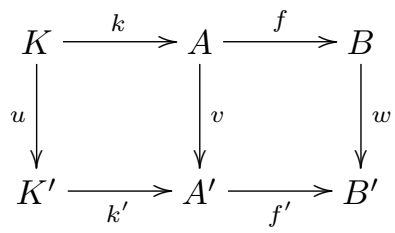

The left-hand square is a pullback if and only if $w$ is a monomorphism.

Proof. If $w$ is a monomorphism, then the left-hand square is clearly a pullback in any quasi-pointed category. On the other hand, when the left-hand square is a pullback, $k$ is the kernel of $f^{\prime} \circ v=w \circ f$. Since in $\mathcal{A}$ the regular epimorphism $f$ is the cokernel of its kernel, it follows that $w \circ f$ is the regular epi-mono factorization of $f^{\prime} \circ v$, and $w$ is a monomorphism.

Many classical homological lemmas valid in an abelian category still hold in the present more general context; this is the case for the $3 \times 3$-Lemma and for the Snake Lemma, as proved by Bourn in [5]. The following result is known as the First Noether Isomorphism Theorem; it essentially follows from the $3 \times 3$-Lemma (see Theorem 4.3 .10 in $[\mathbf{3}])$ :

Theorem 2.2. Let $k: K \rightarrow A$ and $h: H \rightarrow A$ be two normal monomorphisms of $A$, with an arrow $i: K \rightarrow H$ such that $h \circ i=k$. Then

1. the monomorphism $i: K \rightarrow H$ is normal;

2. there is an induced normal monomorphism $\frac{H}{K} \rightarrow \frac{A}{K}$; and

3. there is an isomorphism $\frac{\frac{A}{K}}{\frac{H}{K}} \cong \frac{A}{H}$. 
Proof. Consider the following diagram

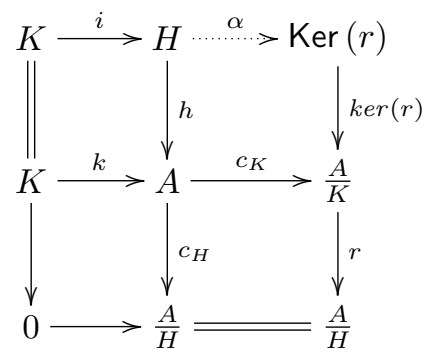

where $c_{H}$ and $c_{K}$ are the canonical quotients, $r$ is the regular epimorphism induced by the inclusion $i$ of $K$ in $H$ and $\alpha$ is the unique arrow such that $k e r(r) \circ \alpha=c_{K} \circ h$. Note that the (unique) arrow from $K$ to 0 exists because $k$ is a kernel by assumption, and that this arrow is a split epimorphism. It follows that the three columns and the two lower rows are short exact sequences, so that the first row is a short exact sequence by the $3 \times 3$-Lemma, and $\operatorname{Ker}(r) \cong \frac{H}{K}$, proving the theorem.

The following well known property of semi-abelian categories [24] still holds in the present quasi-pointed context (see also [32]). From now on, we assume that $\mathcal{A}$ also has the property that any equivalence relation is effective (the kernel pair of a morphism in the category). A regular category which has this property is called Barr-exact $[\mathbf{2}]$.

Lemma 2.3. Consider a commutative square in $\mathcal{A}$ with $k: K \rightarrow A$ a kernel and $q: A \rightarrow B$ a regular epimorphism:

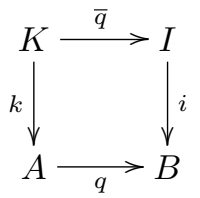

Then the regular image $i: I \rightarrow B$ of $q \circ k$ is a kernel.

Proof. Since by assumption $k$ is a kernel and $\bar{q}$ is a regular epimorphism, it is easy to see that there is also an arrow from $I$ to 0 . Let then $f$ be the cokernel of $k$, and $g$ the pushout of $f$ along $q$ (it exists since the category is exact Mal'tsev [7]):



It is easy to check that the right-hand square is a commutative square with the property that the canonical arrow $\alpha: I \rightarrow \operatorname{Ker}(g)$ to the kernel of $g$ is a regular 
epimorphism: this essentially follows from the fact that the front square in the cube above has the property that the canonical arrow from $A$ to the pullback $Q \times{ }_{Q^{\prime}} B$ is a regular epimorphism (see Theorem 5.7 in [7]). Since $\alpha$ is trivially a monomorphism (the arrow $i$ being a monomorphism) it can be concluded that $i$ is the kernel of $g$.

\section{Birkhoff subcategories}

Recall from $[\mathbf{2 2}]$ that a Birkhoff subcategory of an exact category $\mathcal{A}$ is a full and reflective subcategory $\mathcal{B}$ of $\mathcal{A}$ which is closed in $\mathcal{A}$ under subobjects and quotient objects. Let us write $I: \mathcal{A} \rightarrow \mathcal{B}$ for the reflection and $\eta$ for the unit of the adjunction.

Suppose, from now on, that $\mathcal{A}$ is a finitely complete, quasi-pointed, exact and protomodular category and $\mathcal{B}$ a fixed Birkhoff subcategory of $\mathcal{A}$.

The kernel of $\eta$ (a subfunctor of $1_{\mathcal{A}}: \mathcal{A} \rightarrow \mathcal{A}$ ) will be denoted by $[\cdot]_{\mathcal{B}}: \mathcal{A} \rightarrow \mathcal{A}$, or, when confusion is unlikely to occur, by [.]. We shall sometimes refer to it as the subfunctor associated with $\mathcal{B}$. Remark that, when $\mathcal{A}$ is a variety, the notion of Birkhoff subcategory coincides with the one of subvariety. Consequently, in this case, the associated subfunctor is a subvariety subfunctor.

It has been shown in [22] that for every regular epimorphism $f: A \rightarrow B$ in $\mathcal{A}$ the induced commutative square

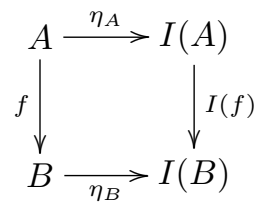

is a pushout. Since all of its arrows are regular epimorphisms, it then follows from Lemma 1.1 of $[6]$ that $[f]$ is a regular epimorphism. Consequently, [·] preserves regular epimorphisms.

For each morphism $f: A \rightarrow B$ in $\mathcal{A}$, we write $R[f]$ for the kernel pair of $f$. Clearly, $[R[f]]$ is a reflexive relation on $[A]$, hence $[R[f]]$ is an effective equivalence relation on $[A]$.

Definition 3.1. For any regular epimorphism $f: A \rightarrow B$ in $\mathcal{A}$, we define $[\operatorname{Ker}(f), A]_{\mathcal{B}}$ as the composite $\left[\pi_{2}\right] \circ k$, where $k$ is the kernel of $\left[\pi_{1}\right]$ :

$$
\operatorname{Ker}\left(\left[\pi_{1}\right]\right) \underset{k}{\longrightarrow}[R[f]] \underset{\left[\pi_{2}\right]}{\stackrel{\left[\pi_{1}\right]}{\longrightarrow}}[A] .
$$

The arrow $\left[\pi_{2}\right] \circ k$ is the normalization of the congruence $[R[f]]$.

We shall often write $[\operatorname{Ker}(f), A]$ instead of $[\operatorname{Ker}(f), A]_{\mathcal{B}}$ and we shall sometimes refer to it as to the commutator associated with $\mathcal{B}$. Also, if $N$ is a normal subobject of an object $A \in \mathcal{A}$ and $c_{N}: A \rightarrow \frac{A}{N}$ is the canonical quotient, then we shall sometimes write $[N, A]$ instead of $\left[\operatorname{Ker}\left(c_{N}\right), A\right]$.

Remark that $[\operatorname{Ker}(f), A]$ is the kernel of the coequalizer of $\left[\pi_{1}\right]$ and $\left[\pi_{2}\right]$ (by $[\mathbf{5}]$ Corollary 6), and it coincides with the definition of $V_{1} f$ by Everaert and Van der Linden in the context of semi-abelian categories $[\mathbf{1 1}]$. 
One says that an extension (a regular epimorphism) $f: A \rightarrow B$ in $\mathcal{A}$ is a central extension if $[\operatorname{Ker}(f), A]=0$. As shown in [6], this definition coincides with the categorical notion of central extension in $\mathcal{A}$ with respect to the Birkhoff subcategory $\mathcal{B}$ introduced by Janelidze and Kelly in $[\mathbf{2 2}]$.

By definition, the subobject $\left[\pi_{2}\right] \circ k$ is normal in $[A]$. We are now going to explain why $\operatorname{ker}\left(\eta_{A}\right) \circ\left[\pi_{2}\right] \circ k$ is also a normal subobject of $A$. One can easily check that $[\operatorname{Ker}(f), A] \cong \pi_{2}\left(\operatorname{Ker}\left(\pi_{1}\right) \wedge[R[f]]\right)$ and that $\operatorname{Ker}\left(\pi_{1}\right) \wedge[R[f]]$ is a normal subobject of $R[f]$ as an intersection of two normal subobjects. Hence, by Lemma $2.3, \pi_{2}\left(\operatorname{Ker}\left(\pi_{1}\right) \wedge[R[f]]\right)$ is a normal subobject of $A$. We could then finally define $[\operatorname{Ker}(f), A]$ as the subobject of $A$, namely

$$
[\operatorname{Ker}(f), A]=\left(\operatorname{Ker}\left(\pi_{1}\right), \operatorname{ker}\left(\eta_{A}\right) \circ\left[\pi_{2}\right] \circ k\right) .
$$

Proposition 3.2. If a commutative square of regular epimorphisms in $\mathcal{A}$

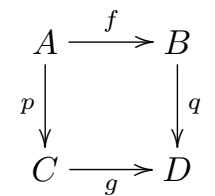

is a pushout, then the induced arrow $[\operatorname{Ker}(f), A] \rightarrow[\operatorname{Ker}(g), C]$ is a regular epimorphism.

Proof. Since, by assumption, the above square is a pushout of regular epimorphisms, the induced morphism $R(p): R[f] \rightarrow R[g]$ is a regular epimorphism (see [7], Theorem 5.7). Since the functor [.] preserves regular epimorphisms, both commutative squares in the following diagram are pushouts of regular epimorphisms:

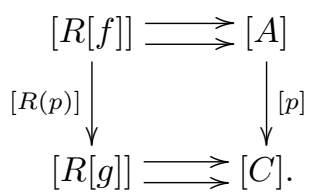

It then follows from Lemma 1.1 in $[6]$ that $[\operatorname{Ker}(f), A] \rightarrow[\operatorname{Ker}(g), C]$ is a regular epimorphism.

\section{Hopf formula and Stallings-Stammbach sequence}

In this section, we define a first and a second homology object by establishing the invariance of the Hopf formula in our context. We then prove the existence of the Stallings-Stammbach exact sequence associated with any short exact sequence.

By projective presentation of $A$ we mean a (regular) projective object $P$ and a normal subobject $R$ of $P$ with

$$
R \longrightarrow P \longrightarrow A
$$

a short exact sequence such that $A \cong \frac{P}{R}$.

A category $\mathcal{A}$ has enough projectives if for every object $A \in \mathcal{A}$ there exist both a projective object $P$ and a regular epimorphism $p: P \rightarrow A$. 
From now on, we shall always assume that the category $\mathcal{A}$ is

- finitely complete

- quasi-pointed

- exact

- protomodular

and furthermore, that

- $\mathcal{A}$ has enough projectives.

Let $\operatorname{Pr} \mathcal{A}$ denote the category of projective presentations of objects in $\mathcal{A}$. An arrow $(r, p, f)$ in $\operatorname{Pr} \mathcal{A}$ between projective presentations $A \cong \frac{P}{R}$ and $B \cong \frac{Q}{S}$ is a commutative diagram

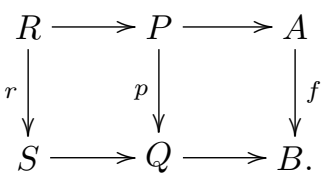

A functor $F: \operatorname{Pr} \mathcal{A} \rightarrow \mathcal{A}$ will be called a Baer invariant if $f=f^{\prime}$ implies $F(r, p, f)=$ $F\left(r^{\prime}, p^{\prime}, f^{\prime}\right)$, for any two arrows $(r, p, f)$ and $\left(r^{\prime}, p^{\prime}, f^{\prime}\right)$ between projective presentations $A \cong \frac{P}{R}$ and $B \cong \frac{Q}{S}$.

The following proposition establishes the invariance of the Hopf formula in our categorical context.

Proposition 4.1. The functor that maps a projective presentation $A \cong \frac{P}{R}$ to the quotient $\frac{R \wedge[P]}{[R, P]}$ is a Baer invariant. In particular, the expression $\frac{R \wedge[P]}{[R, P]}$ is independent of the projective presentation $A \cong \frac{P}{R}$.

Proof. It is easily verified that $[R, P] \leqslant R \wedge[P]$, i.e. as a subobject of $P,[R, P]$ factors through $R \wedge[P]$. Moreover, since $[R, P]$ is defined as a kernel, its inclusion in $R \wedge[P]$ is normal.

Suppose that $(r, p, f)$ and $\left(r^{\prime}, p^{\prime}, f\right)$ are arrows between projective presentations $A \cong \frac{P}{R}$ and $B \cong \frac{Q}{S}$. We are going to prove that $(r, p, f)$ and $\left(r^{\prime}, p^{\prime}, f\right)$ induce the same arrow $\frac{R \wedge[P]}{[R, P]} \rightarrow \frac{S \wedge[Q]}{[S, Q]}$. For this, consider the arrows $[p]$ and $\left[p^{\prime}\right]$, the canonical quotients $c_{[P]}:[P] \rightarrow \frac{[P]}{[R, P]}$ and $c_{[Q]}:[Q] \rightarrow \frac{[Q]}{[S, Q]}$, and the induced arrows $c([p])$ and $c\left(\left[p^{\prime}\right]\right)$ making the obvious squares in the following diagram commute:

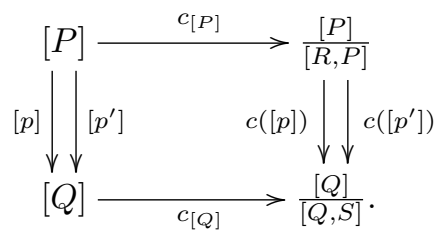

Consider, furthermore, the canonical quotients $c_{P}: P \rightarrow A$ and $c_{Q}: Q \rightarrow B$. Since

$$
c_{Q} \circ p=c_{Q} \circ p^{\prime},
$$


also

$$
c_{[Q]} \circ[p]=c_{[Q]} \circ\left[p^{\prime}\right]
$$

hence $c([p])=c\left(\left[p^{\prime}\right]\right)$.

Let us consider another commutative diagram in which the horizontal arrows are induced by the inclusions $[R, P] \leqslant R \wedge[P]$ and $[S, Q] \leqslant S \wedge[Q]$, respectively, and the left hand downward pointing arrows by $p$ and $p^{\prime}$.

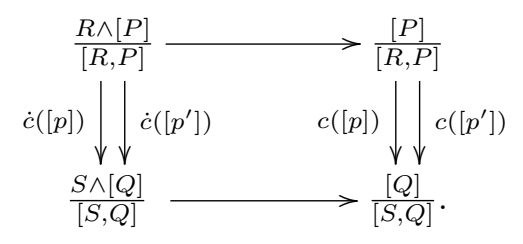

By Proposition 2.1, the horizontal arrows are monomorphisms. Hence, $c([p])=$ $c\left(\left[p^{\prime}\right]\right)$ implies that $\dot{c}([p])=\dot{c}\left(\left[p^{\prime}\right]\right)$.

In order to see that the expression $\frac{R \wedge[P]}{[R, P]}$ is independent of the projective presentation $A \cong \frac{P}{R}$ of $A$, consider another projective presentation $A \cong \frac{Q}{S}$. Since $P$ and $Q$ are projective, there exist arrows $p: P \rightarrow Q$ and $q: Q \rightarrow P$ such that $c_{Q} \circ p=c_{P}$ and $c_{P} \circ q=c_{Q}$. Consequently, there exists an endomorphism $\left(r, q \circ p, 1_{A}\right)$ on $A \cong \frac{P}{R}$. Since also $\left(1_{R}, 1_{P}, 1_{A}\right)$ is an endomorphism on $A \cong \frac{P}{R}$, we have

$$
\dot{c}([q \circ p])=\dot{c}\left(\left[1_{P}\right]\right)=1_{\frac{R \wedge[P]}{[R, P]}} .
$$

Similarly, $\dot{c}([p \circ q])=1_{\frac{S \wedge[Q]}{[S, Q]}}$, hence

$$
\frac{R \wedge[P]}{[R, P]} \cong \frac{S \wedge[Q]}{[S, Q]}
$$

Let us consider a Birkhoff subcategory $\mathcal{B}$ of $\mathcal{A}$, and let $I$ be the reflection $\mathcal{A} \rightarrow \mathcal{B}$.

Definition 4.2. $\quad$ 1. For any object $A \in \mathcal{A}$ we define $H_{1}(A)=I(A)=\frac{A}{[A]}$ the first homology object of $A$;

2. for a projective presentation $A \cong \frac{P}{R}$, the quotient $\frac{R \wedge[P]}{[R, P]}$ will be denoted $H_{2}(A)$, and it will be called the second homology object of $A$.

Remark 4.3. By Proposition 4.1, $H_{2}$ defines, up to natural isomorphism, a functor $\mathcal{A} \rightarrow \mathcal{A}$. More precisely, each choice of projective presentations of the objects of $\mathcal{A}$ yields a functor $H_{2}: \mathcal{A} \rightarrow \mathcal{A}$, and any two such functors are naturally isomorphic.

Remark 4.4. Van der Linden recently proved that the above definition of the second homology object coincides with the second cotriple homology object (in the sense of Barr and Beck [1]) whenever $\mathcal{A}$ is monadic over Set ([32], Theorem 4.2.4).

Lemma 4.5. For any projective presentation $A \cong \frac{P}{R}$, the sequence

$$
0 \longrightarrow H_{2}(A) \longrightarrow \frac{[P]}{[R, P]} \longrightarrow A \longrightarrow H_{1}(A)
$$

is an exact sequence; moreover, $A \rightarrow H_{1}(A)$ is a regular epimorphism. 
Proof. From Theorem 2.2 it follows that

$$
H_{2}(A) \longrightarrow \frac{[P]}{[R, P]} \longrightarrow \frac{[P]}{R \wedge[P]}
$$

is a short exact sequence.

Now consider the following diagram:

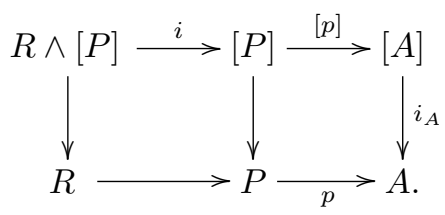

The left hand square is a pullback, by construction. Since $i_{A}$ is a monomorphism and the lower row is a short exact sequence, it follows that $i=k \operatorname{kr}([p])$. The arrow $[p]$ is a regular epimorphism, hence it is the cokernel of its kernel, thus $[A] \cong \frac{[P]}{R \wedge[P]}$. Consequently,

$$
\frac{[P]}{R \wedge[P]} \longrightarrow A \longrightarrow H_{1}(A)
$$

is a short exact sequence.

By pasting these two short exact sequences together, one gets the required exact sequence. The second statement is obvious.

Theorem 4.6. Any short exact sequence

$$
K \stackrel{k}{\longrightarrow} A \stackrel{f}{\longrightarrow} C
$$

yields a five-term exact sequence

$$
H_{2}(A) \stackrel{H_{2}(f)}{\longrightarrow} H_{2}(C) \longrightarrow \frac{K}{[K, A]} \longrightarrow H_{1}(A) \stackrel{H_{1}(f)}{\longrightarrow} H_{1}(C),
$$

with $H_{1}(f)$ a regular epimorphism. This exact sequence depends naturally on the given short exact sequence.

Proof. We can choose projective presentations $A \cong \frac{P}{R}$ and $C \cong \frac{Q}{S}$ such that $P=Q$ and $R \leqslant S$. Then $[R, P] \leqslant[S, P]$ and, by applying Theorem 2.2 , we get the following commutative diagram of short exact rows, where $\beta$ and $\gamma$ are induced by the inclusion $[P] \rightarrow P$ :

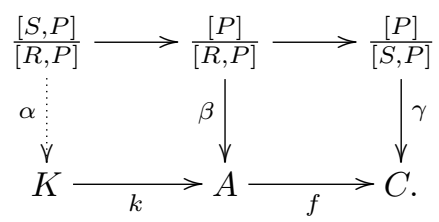

Note that $\alpha$ is induced by the universal property of the kernel $k$, but also by the canonical quotient $c_{R}: P \rightarrow \frac{P}{R}$.

If we prove that $[K, A]$ is the regular image of $\alpha$, the theorem follows from the Snake Lemma ([5], Proposition 14) and Lemma 4.5. 
Clearly, $c_{R}(S)=K$. From Proposition 3.2 it then follows that $c_{R}([S, P])=[K, A]$ and the image of $\alpha$ is $[K, A]$, as desired. The naturality of the five-term sequence is easy to check, and we leave the proof to the reader.

\section{Central series}

In this section we define the lower central series with respect to a Birkhoff subcategory $\mathcal{B}$ and we extend a classical group theoretic result due to Stallings [30]. Our general assumptions allow one to include also a more recent result due to Conduché and Ellis in the context of precrossed modules [8].

As before, we suppose that $\mathcal{A}$ is a quasi-pointed, exact and protomodular category with enough projectives and $\mathcal{B}$ is a fixed Birkhoff subcategory of $\mathcal{A}$.

Definition 5.1. The lower central series of an object $A \in \mathcal{A}$ is the descending sequence

$$
A^{0} \geqslant A^{1} \geqslant \cdots \geqslant A^{n} \geqslant \cdots
$$

defined by putting $A^{0}=A, A^{1}=[A]$ and, for any integer $n \geqslant 1, A^{n+1}=\left[A^{n}, A\right]$.

Note that we could not define $A^{1}$ as $[A, A]$ because $1_{A}$ is not necessarily a kernel, e.g. $1 \rightarrow 1$ can only be a kernel if $\mathcal{A}$ is pointed. However whenever $1_{A}$ is a kernel, we have $[A, A]=[A]$ :

Lemma 5.2. Suppose $A \in \mathcal{A}$ is such that an arrow $A \rightarrow 0$ exists; then $[A, A]=[A]$.

Proof. Of course, the composite

$$
[A] \stackrel{\left[i_{2}\right]}{\longrightarrow}[A \times A] \stackrel{\left[\pi_{1}\right]}{\longrightarrow}[A]
$$

factors through 0 (where $i_{2}=\left(0_{A}, 1_{A}\right)$ ); hence $\left[i_{2}\right]$ factors through $\operatorname{ker}\left(\left[\pi_{1}\right]\right)=$ $[A, A]$. Consequently $\left[\pi_{2}\right] \circ \operatorname{ker}\left(\left[\pi_{1}\right]\right)$ is a split epimorphism:

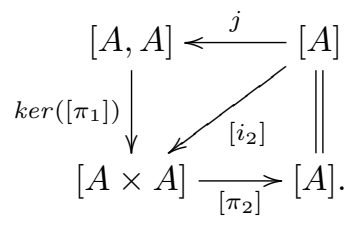

Moreover, since it is the normalization of an equivalence relation, it is also a monomorphism; hence, it is an isomorphism. Consequently $j$ is an isomorphism as well.

Theorem 5.3. Let $f: A \rightarrow B$ be a morphism in $\mathcal{A}$. If

1. the morphism $H_{1}(A) \rightarrow H_{1}(B)$ is an isomorphism;

2. the morphism $\mathrm{H}_{2}(A) \rightarrow \mathrm{H}_{2}(B)$ is a regular epimorphism.

then, for any $n \geqslant 1$, the induced morphism $\frac{A}{A^{n}} \rightarrow \frac{B}{B^{n}}$ is an isomorphism. 
Proof. We prove the theorem by induction. The case $n=1$ is the first assumption. Let us then assume that the property holds for $n$, and we shall prove that it also holds for $n+1$. By assumption, there is a commutative diagram with short exact rows in $\mathcal{A}$ :

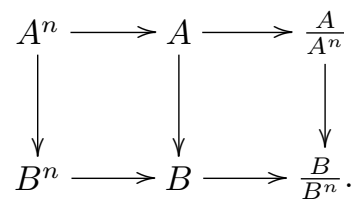

By applying Theorem 4.6 (with $K=A^{n}$ and $C=A / A^{n}$ ), one can show that the left hand vertical arrow in the following diagram with short exact rows is an isomorphism:

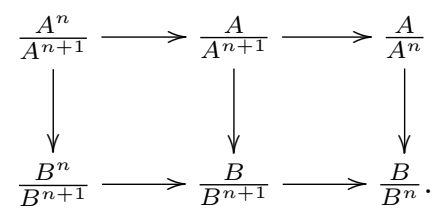

Since the right hand vertical arrow is an isomorphism by the inductive assumption, we can apply the Short Five Lemma and conclude that the central vertical arrow is also an isomorphism.

\section{Examples}

\subsection{Abelian objects}

Recall that an object $A$ in a category $\mathcal{A}$ is abelian if it can be endowed with an internal group structure. In our context, if such a group structure exists, it is necessarily unique. Moreover the subcategory $\operatorname{Ab}(\mathcal{A})$ of $\mathcal{A}$ of all abelian objects is a Birkhoff subcategory.

Let us denote by $\nabla_{A}$ and $\Delta_{A}$ the largest and smallest equivalence relations on an object $A$ respectively. The central extensions in $\mathcal{A}$ with respect to $\operatorname{Ab}(\mathcal{A})$ have been characterized in terms of Smith's commutator of universal algebra $[\mathbf{2 3}][\mathbf{1 7}]$ : an extension $f: A \rightarrow B$ is central if and only if $\left[R[f], \nabla_{A}\right]=\Delta_{A}$. In particular, an object $A \in \mathcal{A}$ is abelian if and only if $\left[\nabla_{A}, \nabla_{A}\right]=\Delta_{A}$.

It is well known that if $\mathcal{A}$ is the variety of groups, Smith's commutator corresponds to the classical group commutator in the following sense: suppose $A$ is a group and $M$ and $N$ are normal subgroups of $A$. Let us denote the group commutator of $M$ and $N$ by $[M, N]$, and the equivalence relations associated with $M$ and $N$ by $R\left[c_{M}\right]$ and $R\left[c_{N}\right]$, respectively. Then $[M, N]$ is the normalization of the equivalence relation $\left[R\left[c_{M}\right], R\left[c_{N}\right]\right]$ on $A$.

Let $\mathcal{A}$ satisfy the conditions of Section 4 . The same proof as that of Theorem 4.4 in [9] shows that sending an extension $f: A \rightarrow B$ in $\mathcal{A}$ to the induced arrow $I(f)=$ $\frac{A}{[\operatorname{Ker}(f), A]} \rightarrow B$ defines a reflection $I: \operatorname{Ext}(\mathcal{A}) / B \rightarrow \operatorname{CExt}(\mathcal{A}) / B$, where $\operatorname{Ext}(\mathcal{A}) / B$ is the category of extensions of $B, \operatorname{CExt}(\mathcal{A}) / B$ is its full subcategory, whose objects are the extensions of $B$ that are central with respect to the Birkhoff subcategory 
$\mathcal{B}$ of $\mathcal{A}$, and $[\operatorname{Ker}(f), A]$ is the commutator associated with $\mathcal{B}$. Furthermore, the functor $I^{\prime}: \operatorname{Ext}(\mathcal{A}) / B \rightarrow \operatorname{CExt}(\mathcal{A}) / B$ which sends an extension $f: A \rightarrow B$ in $\mathcal{A}$ to the induced arrow $I^{\prime}(f): \frac{A}{\left[R[f], \nabla_{A}\right]} \rightarrow B$ is a reflection as well. This is a consequence of the aforementioned characterization of central extensions. Consequently, $I=I^{\prime}$ when $\mathcal{B}=\operatorname{Ab}(\mathcal{A})$ and for any $f: A \rightarrow B$ in $\mathcal{A}$, $[\operatorname{Ker}(f), A]$ is the normalization of the equivalence relation $\left[R[f], \nabla_{A}\right]$ on $A$. In particular, $[A]$ is the normalization of the equivalence relation $\left[\nabla_{A}, \nabla_{A}\right]$ on $A$.

Suppose once more that $\mathcal{A}$ is the variety of groups. It is well known that in this case $\operatorname{Ab}(\mathcal{A})$ is the subvariety of abelian groups. By the above, the associated commutator is the classical commutator of groups. Consequently, in this situation, the results in the present paper imply the classical results due to Stallings [30].

\subsection{Nilpotent objects}

Let us call an object $A \in \mathcal{A}$ nilpotent of class $k$ with respect to a Birkhoff subcategory $\mathcal{B}$ of $\mathcal{A}$ if in the lower central series, $A^{k}=0$. It is readily seen that for each $k \in \mathbb{N}^{*}$, the full subcategory of $\mathcal{A}$ of nilpotent objects of class $k$ is a Birkhoff subcategory. Hence, for any Birkhoff subcategory $\mathcal{B}$, this provides us with, among other things, a whole new array of invariances (Proposition 4.1) and exact sequences (Theorem 4.6).

We consider here only one application, choosing for $\mathcal{A}$ the variety CRng of commutative rings (not necessarily with unit). If $I$ and $J$ are ideals of a commutative ring $R$, then we denote the product

$$
\left\{\sum_{n=1}^{p} i_{n} j_{n} \mid p \in \mathbb{N}, i_{n} \in I, j_{n} \in J, \forall n \leqslant p\right\}
$$

by $I J$. Let us consider the subvariety $0-\mathrm{Rng} \subseteq \mathrm{CRng}$ of zero rings, i.e. those rings $R$ for which $R^{2}=R R=0$. The reflection of CRng into $0-$ Rng sends a commutative ring $R$ to the quotient $\frac{R}{R^{2}}$; consequently $[R]\left(=[R]_{0-\text { Rng }}\right)=R^{2}$. Suppose $I$ is an ideal of a ring $R \in \mathrm{CRng}$. Let us denote by $c_{I}$ the quotient $R \rightarrow \frac{R}{I}$, and its kernel congruence by $R\left[c_{I}\right]$. The commutator $[I, R]=[I, R]_{0-\text { Rng }}$ is defined as the normalization of the congruence $\left(R\left[c_{I}\right]\right)^{2}$ on $R^{2}$. Since

$$
\begin{aligned}
& R\left[c_{I}\right]^{2}=\left\{\sum_{n=1}^{p}\left(r_{n}, r_{n}+i_{n}\right)\left(r_{n}^{\prime}, r_{n}^{\prime}+i_{n}^{\prime}\right) \mid p \in \mathbb{N}, r_{n}, r_{n}^{\prime} \in R, i_{n}, i_{n}^{\prime} \in I, \forall n \leqslant p\right\} \\
= & \left\{\sum_{n=1}^{p}\left(r_{n} r_{n}^{\prime}, r_{n} r_{n}^{\prime}+r_{n} i_{n}^{\prime}+i_{n} r_{n}^{\prime}+i_{n} i_{n}^{\prime}\right) \mid p \in \mathbb{N}, r_{n}, r_{n}^{\prime} \in R, i_{n}, i_{n}^{\prime} \in I, \forall n \leqslant p\right\},
\end{aligned}
$$

one has

$$
[I, R]=\left\{\sum_{n=1}^{p}\left(r_{n} i_{n}^{\prime}+i_{n} r_{n}^{\prime}+i_{n} i_{n}^{\prime}\right) \mid p \in \mathbb{N}, r_{n}, r_{n}^{\prime} \in R, i_{n}, i_{n}^{\prime} \in I, \forall n \leqslant p\right\}=I R .
$$

Consequently for any commutative ring $R$, we find the lower central series

$$
R \geqslant R R \geqslant R R R \geqslant \cdots \text {. }
$$

Let us now consider the subvariety of CRng given by the nilpotent rings of class 2 . 
Denote by $[\cdot]_{2}$ the associated subfunctor and by $[\cdot, \cdot]_{2}$ the associated commutator. By the above, $[R]_{2}=R^{3}$, for any $R \in \mathrm{CRng}$. Hence, for any ideal $I$ of $R \in \mathrm{CRng},[I, R]_{2}$ is defined as the normalization of the congruence $\left(R\left[c_{I}\right]\right)^{3}$ on $R^{3}$. From calculations similar to the ones above, it can be deduced that $[I, R]_{2}=I R^{2}$.

Let us now denote, for any $n \geqslant 1$, the subfunctor and the commutator associated with the subvariety of nilpotent objects of class $n$ by $[\cdot]_{n}$ and $[\cdot, \cdot]_{n}$, respectively. For any commutative ring $R$ and any ideal $I$ of $R$, it is found that $[R]_{n}=R^{n+1}$ and $[I, R]_{n}=I R^{n}$.

Hence we obtain, for instance from Proposition 4.1, that for any ring $R \in \mathrm{CRng}$ and each $n \geqslant 1$, the expression

$$
\frac{I \wedge P^{n+1}}{I P^{n}}
$$

is independent of the projective presentation $I \rightarrow P \rightarrow R$. If, furthermore, $J \rightarrow Q \rightarrow S$ is a projective presentation of a commutative ring $S$, and

$$
K \rightarrow R \rightarrow S
$$

a short exact sequence, we obtain from Theorem 4.6 an exact sequence

$$
\frac{I \wedge P^{n+1}}{I P^{n}} \rightarrow \frac{J \wedge Q^{n+1}}{J Q^{n}} \rightarrow \frac{K}{K R^{n}} \rightarrow \frac{R}{R^{n+1}} \rightarrow \frac{S}{S^{n+1}} \rightarrow 0
$$

for any $n \geqslant 1$.

In his thesis [15], Furtado-Coelho considered a similar situation to the one above, by choosing for $\mathcal{A}$ the variety $\mathrm{Gp}$ of groups. For any $k \in \mathbb{N}^{*}$, let us denote by $\mathrm{Nil}_{k}$ the subvariety of $k$-nilpotent groups in the variety Gp. Proposition 3.3.1 of [15] states, in particular, the following (see also Corollary 3.5 in [10]): for any group $A$ and any normal subgroup $N$ of $A$,

1. $[N, A]_{\mathrm{Nil}_{0}}=N$;

2. $[N, A]_{\mathrm{Nil}_{k+1}}=\left[[N, A]_{\mathrm{Nil}_{k}}, A\right]$ for any $k \in \mathbb{N}^{*}$,

where the last commutator is the ordinary commutator of groups. Hence, for instance, Proposition 4.1 implies that, for any group $A$, the expression

$$
\frac{R \wedge[[P, P], P]}{[[R, P], P]}
$$

is independent of the projective presentation $R \rightarrow P \rightarrow A$.

\subsection{Internal precrossed modules}

An important class of examples of quasi-pointed, exact and protomodular categories is given by the categories $\operatorname{RG}(\mathcal{A}) / B$ of reflexive graphs over a fixed object $B$ in an exact and protomodular category $\mathcal{A}$. A reflexive graph $(A, d, c, i)$ consists of an object $A$, three arrows $d, c: A \rightarrow B$ and $i: B \rightarrow A$, such that $d \circ i=1_{B}=c \circ i$. A morphism $f:(A, d, c, i) \rightarrow\left(C, d^{\prime}, c^{\prime}, i^{\prime}\right)$ of reflexive graphs over $B$ is an arrow in 
$\mathcal{A}$ making the three obvious triangles commutative:

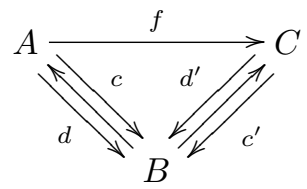

Since limits and colimits (of non-empty diagrams) in $\operatorname{RG}(\mathcal{A}) / B$ are just limits and colimits in $\mathcal{A}$, it is readily seen that $\operatorname{RG}(\mathcal{A}) / B$ is also exact and protomodular. Moreover, since the unique morphism $0=\left(B, 1_{B}, 1_{B}, 1_{B}\right) \rightarrow\left(B \times B, \pi_{1}, \pi_{2}, \delta\right)=1$ is a monomorphism, $\operatorname{RG}(\mathcal{A}) / B$ is quasi-pointed.

It is well known that the category $\mathrm{RG}(\mathrm{Gp}) / B$ of reflexive graphs of groups over a fixed group $B$ is equivalent to $B-\operatorname{PrCM} / B$, the category of precrossed $B$-modules. Moreover, via this equivalence, the category $\mathrm{Gpd}(\mathrm{Gp}) / B$ corresponds to the category $B-\mathrm{CM}$ of crossed $B$-modules:

$$
\begin{gathered}
\mathrm{RG}(\mathrm{Gp}) / B \simeq B-\operatorname{PrCM} \\
\cup \quad \cup \\
\mathrm{Gpd}(\mathrm{Gp}) / B \simeq B-\mathrm{CM} .
\end{gathered}
$$

When $B$ is a group, recall that a precrossed $B$-module $(M, \partial)$ is a group homomorphism $\partial: M \rightarrow B$ provided with a (left) action of $B$ on $M$ such that

$$
\partial\left({ }^{b} m\right)=b \partial(m) b^{-1}
$$

for all $b \in B$ and $m \in M .(M, \partial)$ is a crossed $B$-module if, moreover, the Peiffer condition

$$
{ }^{\partial\left(m^{\prime}\right)} m=m^{\prime} m\left(m^{\prime}\right)^{-1}
$$

is satisfied for all $m, m^{\prime} \in M$. A morphism of (pre-)crossed $B$-modules $f:(M, \partial) \rightarrow$ $(N, \epsilon)$ is a group homomorphism $f: M \rightarrow N$ with $\epsilon \circ f=\partial$, which preserves the action.

Similarly, the categories of precrossed $B$-rings and of crossed $B$-rings are equivalent to the categories $\mathrm{RG}(\mathrm{Rng}) / B$ of reflexive graphs in $\mathrm{Rng}$ and $\mathrm{Gpd}(\mathrm{Rng}) / B$ of groupoids in Rng (over a fixed ring $B$ ), respectively [26]. More generally, Janelidze proved in $[\mathbf{2 1}]$ that these equivalences can be extended from groups and rings to any semi-abelian category. Furthermore, in each of these cases $\operatorname{Gpd}(\mathcal{A}) / B$ is a Birkhoff subcategory of $\operatorname{RG}(\mathcal{A}) / B$, hence the results of the present paper can be applied to these situations.

For any Mal'tsev variety $\mathcal{A}$, we characterized in $[\mathbf{9}]$ the central extensions in $\mathrm{RG}(\mathcal{A}) / B$ with respect to $\operatorname{Gpd}(\mathcal{A}) / B$, in terms of the universal algebraic commutator introduced by Smith [29]. More precisely, an extension $f:(A, d, c, i) \rightarrow\left(C, d^{\prime}, c^{\prime}, i^{\prime}\right)$ of reflexive graphs over $B$ (as in the diagram above) is central if and only if

$$
[R[d] \vee R[c], R[f]]=\Delta_{A},
$$

where $\Delta_{A}$ is the smallest congruence on $A$.

Let us now consider, in more detail, the case where $\mathcal{A}$ is the variety of groups. Recall that the Peiffer commutator of two sub-precrossed $B$-modules (subobjects 
in $B-\operatorname{PrCM}) S, T \leqslant M=(M, \partial)$, denoted $\langle S, T\rangle_{G p}$, is the normal subgroup of the group $M$ generated by the elements $\langle s, t\rangle=s t s^{-1}\left({ }^{\partial s} t\right)^{-1}$ and $\langle t, s\rangle=t s t^{-1}\left({ }^{2 t} s\right)^{-1}$ for all $s \in S$ and $t \in T$.

Note that the reflection from $B-\operatorname{PrCM}$ to $B-\mathrm{CM}$ is defined by associating the crossed $B$-module $\frac{M}{\langle M, M\rangle}$ with a precrossed $B$-module $(M, \partial)$. Thus the associated subfunctor sends $(M, \partial)$ to $(\langle M, M\rangle, 0)$ (where 0 denotes the trivial homomorphism $\langle M, M\rangle \rightarrow B)$.

We will now explain why the commutator $[\cdot, \cdot]_{B-\mathrm{CM}}$ associated with the subcategory $B-\mathrm{CM}$ coincides with the Peiffer commutator. More precisely, if $f: A \rightarrow C$ is a regular epimorphism in $B-\operatorname{PrCM}$, then

$$
[\operatorname{Ker}(f), A]_{B-\mathrm{CM}}=(\langle\operatorname{Ker}(f), A\rangle, 0),
$$

where 0 denotes the trivial homomorphism $\langle\operatorname{Ker}(f), A\rangle \rightarrow B$. As mentioned above, the category $B-\operatorname{PrCM}$ is equivalent to $\mathrm{RG}(\mathrm{Gp}) / B$. Let us denote by $\mathcal{P}: \operatorname{RG}(\mathrm{Gp}) / B$ $\rightarrow B-\operatorname{PrCM}$ the corresponding functor.

Suppose $f:(A, d, c, i) \rightarrow\left(C, d^{\prime}, c^{\prime}, i^{\prime}\right)$ is an extension of reflexive graphs in $\mathrm{Gp}$. Let us consider $[R[d] \vee R[c], R[f]]$ as a congruence on the reflexive graph $(A, d, c, i)$ in $\mathrm{RG}(\mathrm{Gp}) / B$. One can verify that via the equivalence $\mathcal{P}$, the normalization of $[R[d] \vee R[c], R[f]]$ corresponds to the Peiffer commutator $\langle\operatorname{Ker}(\mathcal{P}(f)), \mathcal{P}(A)\rangle$. Furthermore, in [9] we proved that the normalization of $[R[d] \vee R[c], R[f]]$ coincides with the commutator $[\operatorname{Ker}(f),(A, d, c, i)]_{\mathrm{Gpd}(\mathrm{Gp}) / B}$. Since, of course, $[\cdot, \cdot]_{\mathrm{Gpd}(\mathrm{Gp}) / B}$ corresponds, via $\mathcal{P}$, to $[\cdot, \cdot]_{B-\mathrm{CM}}$, we find that $[\cdot, \cdot]_{B-\mathrm{CM}}$ coincides with the Peiffer commutator.

Note that $B-\operatorname{PrCM}$ is easily seen to have enough projectives, since it is a manysorted variety (but it is not a one-sorted variety; see [9]). Since pullbacks (resp. pushouts) in $B-\operatorname{PrCM}$ are obtained by taking pullbacks (resp. pushouts) in Gp, and because the initial object of $B-\operatorname{PrCM}$ is $\left(0,0_{B}\right)$ (where $0_{B}$ is the unique homomorphism $0 \rightarrow B$ ), the kernels and cokernels in $B-\operatorname{PrCM}$ correspond to kernels and cokernels in Gp; hence Definition 4.2 yields, in particular, homology groups

$$
H_{1}(M, \partial)=\frac{M}{\langle M, M\rangle} \quad \text { and } \quad H_{2}(M, \partial)=\frac{\operatorname{Ker}(p) \wedge\langle P, P\rangle}{\langle\operatorname{Ker}(p), P\rangle}
$$

(where $p:(P, \partial \circ p) \rightarrow(M, \partial)$ is a projective presentation of $(M, \partial))$ and, for instance, Theorem 4.6 particularizes to the following proposition from $[8]$ :

Corollary 6.1. Any short exact sequence of precrossed B-modules

$$
K \longrightarrow M \longrightarrow N
$$

yields an exact sequence of groups

$$
H_{2}(M) \longrightarrow H_{2}(N) \longrightarrow \frac{K}{\langle K, M\rangle} \longrightarrow H_{1}(M) \longrightarrow H_{1}(N) \longrightarrow 0 .
$$

This exact sequence depends naturally on the given short exact sequence.

Note that if we choose $B=0$, the trivial group, then the Peiffer commutator is just the commutator of groups, and the above exact sequence is nothing but the classical Stallings-Stammbach sequence. 
We would also like to mention that other results similar to the ones above can be obtained in the context of profinite (pre-)crossed modules as defined by Korkes and Porter in [25].

Finally, suppose that $B$ is a ring. Recall from $[\mathbf{2 6}]$ that a precrossed $B$-ring $(R, \partial)$ is a two sided $B$-module together with a $B$-module morphism $\partial: R \rightarrow B$ ( $B$ acts upon itself by left and right multiplication). $(R, \partial)$ is a crossed $B$-ring if, moreover,

$$
\partial(r) r^{\prime}=r r^{\prime}=r \partial\left(r^{\prime}\right)
$$

for all $r, r^{\prime} \in R$. A morphism of (pre-)crossed $B$-rings $f:(R, \partial) \rightarrow(S, \epsilon)$ is a $B$ module morphism $f: R \rightarrow S$ with $\epsilon \circ f=\partial$.

Suppose $I$ and $J$ are two sub-precrossed $B$-rings of a precrossed $B$-ring $R$. We denote by $\langle I, J\rangle_{R n g}$ the ideal of $R$ generated by the elements $\langle i, j\rangle=\partial(i) j-i j$ and $\langle i, j\rangle^{\prime}=i \partial(j)-i j$, for all $i \in I$ and $j \in J$, and call this ideal the Peiffer commutator of $I$ and $J$.

It is then easily verified that, similarly to the case of precrossed modules, Definition 4.2 yields, in particular, homology rings $H_{1}(R, \partial)$ and $H_{2}(R, \partial)$, defined in terms of the Peiffer commutator of rings, for each precrossed $B$-ring $(R, \partial)$. Hence, as in the case of precrossed modules, we obtain, for instance, for any exact sequence in $B$ - PrCRng, an exact sequence of rings in terms of the Peiffer commutator.

\section{References}

[1] M. Barr and J. Beck, Homology and standard constructions, in Seminar on triples and categorical homology theory, Lecture Notes in Mathematics, vol. 80, Springer, 1969, pp. 245-335.

[2] M. Barr, P. A. Grillet, and D. H. van Osdol, Exact categories and categories of sheaves, Lecture Notes in Mathematics, vol. 236, Springer, 1971.

[3] F. Borceux and D. Bourn, Mal'cev, Protomodular, Homological and SemiAbelian Categories, Math. Appl., vol. 566, Kluwer Academic Publishers, 2004.

[4] D. Bourn, Normalization equivalence, kernel equivalence and affine categories, Category Theory, Proceedings Como 1990, Lecture Notes in Mathematics, vol. 1488, Springer, 1991, pp. 43-62.

[5] D. Bourn, $3 \times 3$ lemma and protomodularity, J. Algebra 236 (2001), 778-795.

[6] D. Bourn and M. Gran, Central extensions in semi-abelian categories, J. Pure Appl. Algebra 175 (2002), 31-44.

[7] A. Carboni, G. M. Kelly, and M. C. Pedicchio, Some remarks on Maltsev and Goursat categories, Appl. Categ. Struct. 1 (1993), 385-421.

[8] D. Conduché and G. J. Ellis, Quelques propriétés homologiques des modules précroisés, J. Algebra 123(2) (1989), 327-335.

[9] T. Everaert and M. Gran, Precrossed modules and Galois theory, J. Algebra 297 (2006), 292-309.

[10] T. Everaert and M. Gran, Relative commutator associated with varieties of $n$-nilpotent and of $n$-solvable groups, Arch. Math. (Brno) 42(4) (2006), 387396. 
[11] T. Everaert and T. Van der Linden, Baer invariants in semi-abelian categories I: general theory, Theory Appl. Categ. 12(1) (2004), 1-33.

[12] T. Everaert and T. Van der Linden, Baer invariants in semi-abelian categories II: homology, Theory Appl. Categ. 12(4) (2004), 195-224.

[13] L. Franco, Baer invariants of crossed modules, J. Algebra 160 (1991), 50-56.

[14] A. Fröhlich, Baer-invariants of algebras, Trans. Amer. Math. Soc. 109 (1963), 221-244.

[15] J. Furtado-Coelho, Varieties of $\Omega$-groups and associated functors, Ph.D. dissertation, University of London, 1972.

[16] J. Furtado-Coelho, Homology and generalized Baer invariants, J. Algebra 40 (1976), 596-609.

[17] M. Gran, Applications of categorical Galois theory in universal algebra, in Galois Theory, Hopf Algebras, and Semi-Abelian Categories, Fields Institute Communications Series (2004), pp. 243-280.

[18] M. Gran and T. Van der Linden, On the second cohomology group in semiabelian categories (2005), arxiv:math.KT/0511357.

[19] P. J. Higgins, Groups with multiple operators, Proc. London Math. Soc. (1956), 366-416.

[20] S. A. Huq, Commutator, nilpotency and solvability in categories, Quart. J. Math. Oxford 19(2) (1968), 363-389.

[21] G. Janelidze, Internal crossed modules, Georgian Math. Journal 10 (2003), 99-114.

[22] G. Janelidze and G. M. Kelly, Galois theory and a general notion of central extension, J. Pure Appl. Algebra 97 (1994), 135-161.

[23] G. Janelidze and G. M. Kelly, Central extensions in Mal'tsev varieties, Theory Appl. Categ. 7(10) (2000), 219-226.

[24] G. Janelidze, L. Márki, and W. Tholen, Semi-abelian categories, J. Pure Appl. Algebra 168 (2002), 367-386.

[25] F.J. Korkes and T. Porter, Pro- $C$ completions of crossed modules, Proc. Edinb. Math. Soc., II. Ser. 33(1) (1990), 39-51.

[26] R. Lavendhomme and J.R. Roisin, Cohomologie non abélienne de structures algébriques, J. Algebra 67 (1980), 385-414.

[27] A. S.-T. Lue, Baer-invariants and extensions relative to a variety, Proc. Camb. Phil. Soc. 63 (1967), 569-578.

[28] M. C. Pedicchio, A categorical approach to commutator theory, J. Algebra 177 (1995), 647-657.

[29] J. D. H. Smith, Mal'cev varieties, Lecture Notes in Mathematics, vol. 554, Springer, 1976.

[30] J. Stallings, Homology and central series of groups, J. Algebra 2 (1965), 170181. 
Homology, Homotopy and Applications, vol. 9(1), 2007

[31] U. Stammbach, Anwendungen der Homologietheorie der Gruppen auf Zentralreihen und auf Invarianten von Präsentierungen, Math. Z. 94 (1966), 157177.

[32] T. Van der Linden, Homology and homotopy in semi-abelian categories, Ph.D. Dissertation, Vrije Universiteit Brussel, 2006, arxiv:math.CT/0607100.

Tomas Everaert teveraer@vub.ac.be

Vakgroep Wiskunde

Faculteit Wetenschappen

Vrije Universiteit Brussel

Pleinlaan 2, 1050 Brussel

Belgium

Marino Gran gran@lmpa.univ-littoral.fr

Lab. Math. Pures et Appliquées

Université du Littoral Côte d'Opale

50, Rue F. Buisson

62228 Calais

France

This article is available at http://intlpress.com/HHA/v9/n1/a12 